\title{
State Detection During Paraplegic Gait as Part of a Finite State Based Controller
}

\author{
Henry M. Franken', Student Member, IEEE, Wijbe de Vries', Peter H. Veltink', Member, IEEE, \\ Gert Baardman' and Herman B.K. Boom', Member, IEEE
}

'Biomedical Technological Institute, University of Twente, 7500 AE Enschede, the Netherlands

${ }^{2}$ Roessingh R\&D, $7500 \mathrm{AH}$ Enschede, The Netherlands

Abstract-A finite-state model of paraplegic gait may serve as the basis for the design of an hierarchical control system for locomotion assisted by Functional Electrical Stimulation (FES). This approach compels the control system to detect the different states on-line during gait.

Experimental results of two state detection strategies are presented. The preliminary results include various sensory feedback signals, such as hip gonio, footswitch and crutch force signals. Video recordings provided off-line comparison. State detection on the basis of footswitch and gonio data was problematic, probably due to time varying weight distribution in combination with a switch forcelevel and problems with robust attachment of the footswitches. Gonio together with crutch force data appeared to be more reliable for state detection.

\section{INTRODUCTION}

A hybrid assistive system (HAS) comprising a FES-unit (stimulator, electrodes etc.), an orthosis and crutches may be used to restore gait in paraplegics [1..4]. Such a hybrid system consisting of a reciprogating gait orthosis (RGO) with locked knees and a four-channel portable stimulator was used in the experiments in this paper. In this system, supervisory control of the stimulation was performed by the patient operating finger switches in the crutches.

Manual control or cloned-control of well-trained patients [3] offers poor flexibility of control tasks, optimization of stimulation onset and adjustment feasibilities concerning muscle fatigue $[2,5]$. These disadvantages point out the need for a different kind of supervisory control.

With a high control level, based on a finite state model of paraplegic gait, such a supervisory control seems feasible $[1,3,4]$. However, this approach necessitates robust real time detection of states during gait.

This paper discusses a model of paraplegic gait and presents experimental results of the initial impetus of on-line state detection on the basis of two different sensor sets.

\section{Fintte State Model of Paraplegic GatT}

In our approach, paraplegic gait is modeled as a finite state machine $M$, with $M$ a quintuple

$$
M=\left(S, X, Z, f_{3}, f_{2}\right)
$$

where $S, X$ and $Z$ the internal state, input and output alphabet respectively. In addition, $f_{\text {, }}$ and $f_{z}$ depict the state transition and output function respectively.

With $\sigma_{0}$ the initial state of $M$, the recurrence relations

$$
\begin{aligned}
& s(t+1)=f_{s}(s(t), x(t)) \\
& z(t)=f_{z}(s(t), x(t))
\end{aligned}
$$

with $s \in S, x \in X$ and $z \in Z$, define the operator $T\left(M, \sigma_{0}\right)$ which is the behavior of the initialized machine $\left(M, \sigma_{0}\right)$.

The internal state set $S$ of $M$ comprises 9 different dynamic and quasi-static states which are described in Table 1. State 9 designates potential danger for the patient and is added to the set as a safety measure.

\begin{tabular}{|c|c|}
\hline Table 1. State description of the finite-state machine $M$. \\
\hline 0 & Description \\
\hline 1 & double support; legs alongside \\
\hline 2 & double support; right leg in front \\
\hline 3 & early swing left leg \\
\hline 4 & mid swing left leg \\
\hline 5 & late swing left leg \\
\hline 6 & double support; left leg in front \\
\hline 7 & early swing right leg \\
\hline 8 & mid swing right leg \\
\hline 9 & late swing right leg \\
\hline
\end{tabular}

\section{State Detection Strategres}

\section{A. Switch-Gonio Strategy}

The first detection strategy was based upon the description of the states (Table 1) with gonio and footswitch signals. These descriptions were implemented in software, by means of IF..THEN.. ELSE statements.

Hip gonio signals were measured by two goniometers 
placed upon the pivot point of the hips. This configuration, facilitated the measurement of the angles between upper body and leg (hip-angle). For the software implementation, the hip angle difference $\varphi_{d}$, defined as the right hip angle minus the left hip angle, was used. So, $\varphi_{d}$ is, e.g., positive in state 1 .

In addition, eight footswitches per foot were responsible for floor-contact detection. The situation that both feet were not in contact with the floor, was obviously unstable and therefore referred to as state 9 .

\section{B. Force-Gonio Strategy}

The second strategy required a description of states by means of gonio signals as discussed in above and crutch force signals. The force signals were obtained with piezo electrical transducers inside the crutches. State 9 now corresponded to the situation of both crutch forces simultaneously being below a threshold close to zero.

Again IF..THEN..ELSE statements were used to implement the descriptions in the software.

\section{IV.RESULTS}

Both detection strategies have been verified with sensory feedback of different gait experiments, concerning one paraplegic subject. During these experiments no electrical stimulation was used.

Fig. 1 shows sensory feedback that was used to obtain the state index registration of Fig. 2 according to the force-gonio strategy. The signals in Fig. 1 are part of a trial of $60 \mathrm{~s}$. Video recordings provided objective off-line comparison.

Similar registrations to verify the switch-gonio strategy were recorded. It appeared that floor-contact detection by means of footswitches had a high failure rate. This resulted in a poor state detection with frequent detection of state 9 which was not observed in the video recording.

\section{CONCLUSION AND DISCUSSION}

Two state detection strategies, based on different sensory sets, were discussed and experimentally investigated by offline registrations of sensory feedback and state index.

It appeared from the preliminary experimental results that the performance of the strategy using crutch forces and gonio feedback was superior to the performance of the strategy using footswitch and gonio feedback. Possible reasons for the poor performance of the latter are

- the time varying weight distribution across the feet during locomotion together with the minimum force necessary to press the footswitches

- problems with robust attachment of the footswitches

Before any of the discussed strategies can be used for the hierarchical cycle-to-cycle control strategy, which is currently under development [5], experiments including FES are necessary.

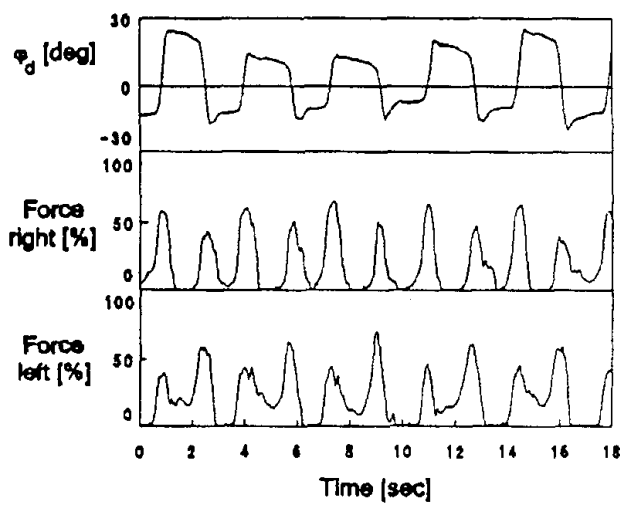

Fig. 1. Registration of the hip-angle difference $\boldsymbol{\Phi}_{\mathrm{d}}$ and the normalized force in the right and left crutch.

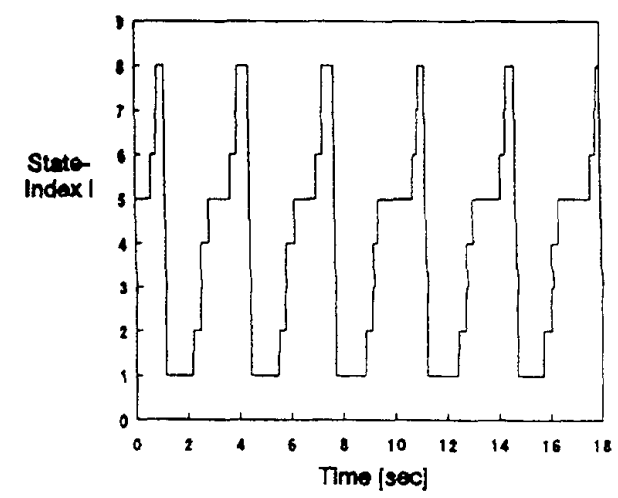

Fig. 2. Registration of different states during locomotion on the basis of crutch-fore and hip-angle difference (Fig. 1).

\section{REFERENCES}

[1] R. Tomović, R. Anastasijevic, J. Vuð̌ and D. Tepavac, The Study of Locomotion by Finite State Models, Biol. Cybern, vol. 63, pp 271-276, 1990.

[2] D. Graupe, K.H. Kohn and S.P. Basses, Control of ElectricallyStimulated Walking of Paraplegics Via Above- and Below-Lesion EMG Signature Identification, IEEE Trans. Aut. Contr., vol. 34, pp 130-137. 1989.

[3] B.J. Andrews, R.B. Bamett, G.F. Phillips, N. Donaldson, D.N. Rushton and T.A. Perkins, Rule-Based Control of a Hybrid FES Orthosis for Assisting Paraplegic Locomotion, Automedica, vol. 11, pp 175-199, 1989.

[4] H.J. Chizeck, R. Kobetic, E.B. Marsolais, J.J. Abbas, Irah H. Donner and E. Simon, Control of Functional Neuromuscular Stimulation Systems for Standing and Locomotion in Paraplegics, Proc. IEEE, vol. 76, pp 1155-1164, 1988.

[5] H.M. Franken, P.H. Veltink, G. Baardman, R.A. Redmeijer, H.B.K. Boom, Experimental On/Off Control of the Swing Phase of Paraplegic Gait Induced by Surface Electrical Stimulation, IEEE EMBS 15th An. Int. Conf., San Diego 1993, These Proceedings. 